\title{
Potential volcanic impacts on future climate variability
}

\author{
Ingo Bethke ${ }^{1 \star}$, Stephen Outten ${ }^{2}$, Odd Helge Otterå', Ed Hawkins ${ }^{3}$, Sebastian Wagner ${ }^{4}$, Michael Sigl ${ }^{5,6}$ \\ and Peter Thorne ${ }^{7}$
}

\begin{abstract}
Volcanic activity plays a strong role in modulating climate variability'. Most model projections of the twenty-first century, however, under-sample future volcanic effects by not representing the range of plausible eruption scenarios ${ }^{2-4}$. Here, we explore how sixty possible volcanic futures, consistent with ice-core records ${ }^{5}$, impact climate variability projections of the Norwegian Earth System Model (NorESM) ${ }^{6}$ under RCP4.5 (ref. 7). The inclusion of volcanic forcing enhances climate variability on annual-to-decadal timescales. Although decades with negative global temperature trends become $\sim 50 \%$ more commonplace with volcanic activity, these are unlikely to be able to mitigate long-term anthropogenic warming. Volcanic activity also impacts probabilistic projections of global radiation, sea level, ocean circulation, and sea-ice variability, the local-scale effects of which are detectable when quantifying the time of emergence ${ }^{8}$. These results highlight the importance and feasibility of representing volcanic uncertainty in future climate assessments.
\end{abstract}

Volcanism has been a major driver of past climate variability ${ }^{1}$ and will continue to affect future climate alongside human influences ${ }^{9}$. Explosive volcanic eruptions warm the stratosphere ${ }^{10}$, cool the troposphere ${ }^{11}$, cause changes in the hydrological cycle $e^{12,13}$, and trigger modifications of atmospheric circulation that give rise to large regional climate responses ${ }^{14}$. The instrumental period covering the past 150 years has been relatively volcanically quiescent, and it is therefore tempting to ascribe potential volcanism a minor role in future climate impact and risk assessments. In a millennial perspective, however, there have been periods with considerably stronger volcanic activity ${ }^{5}$ (Supplementary Fig. 1). Clustered occurrence of strong tropical eruptions has contributed to sustained cold periods such as the Little Ice Age ${ }^{15}$, where the longerterm climate impacts are mediated through ocean heat content anomalies ${ }^{16}$ and ocean circulation changes ${ }^{17-19}$ that also affect global and regional sea level ${ }^{20}$ and sea-ice conditions ${ }^{15,17}$.

Because volcanic eruptions are unpredictable events, they have generally been excluded from twenty-first century climate projection protocols. Most recent projections either specify future volcanic forcing as zero or a constant background value ${ }^{4}$, whereas considerations of more realistic volcanic effects have been limited to idealized eruption scenarios, repeating recent volcanic activity in near-future simulations ${ }^{21,22}$. Herein we explore whether a more complete representation of volcanic forcing uncertainty that considers a range of volcanic forcing possibilities will have an impact on important aspects of probabilistic twenty-first century projections increasingly being used for adaptation planning purposes. The risk from not realistically accounting for volcanic forcing effects is that critical possible future outcomes are being discounted and maladaptation ensues.

The possibility of utilizing stochastic volcanic forcing in projections has been recognized in previous studies ${ }^{23}$ and underscored in the latest assessment report of the Intergovernmental Panel on Climate Change ${ }^{2}$. Increasing computational power, facilitating large ensemble simulations ${ }^{24}$, together with improved reconstructions of past volcanic activity ${ }^{5,25}$ that allow for a better statistical characterization $^{9,23,26}$, make it timely to revisit the question of volcanic effects on twenty-first century climate projections. We start by deriving plausible future volcanic forcings (Fig. 1) by sampling from reconstructed volcanic activity of the past 2,500 years $^{5}$ (Supplementary Fig. 1). We next perform three twenty-first century simulation ensembles with the Norwegian Earth System Model (NorESM) ${ }^{6}$, that use the same mid-range anthropogenic forcing scenario RCP4.5 (ref. 7) but differ in their volcanic forcing: a 60-member ensemble using plausible stochastic volcanic forcing (VOLC); a 60-member reference ensemble using zero volcanic forcing (NO-VOLC); and a 20-member ensemble using 1850-2000 averaged volcanic forcing $^{27}$ (VOLC-CONST). NO-VOLC and VOLC-CONST are the two approaches that were adopted across the group of models contributing twenty-first century projections to the Coupled Model Intercomparison Project phase 5 (CMIP5) (ref. 3). Hence we consider both as useful counterfactual cases here to aid reader interpretation of possible limitations in existing twenty-first century projection runs. Specifically, we assessed the volcanic influence on the climate variability and means of future projections by comparing our three ensembles for several societally relevant diagnostics.

We start by examining the impact of future volcanic activity on Global-Mean Surface Air Temperature (GMST) — an integrated climate-change indicator of particular relevance to mitigation decision making. Figure 2a shows annual-mean GMST changes over the course of the twenty-first century as simulated in the three ensembles. The effect of volcanic forcing on the ensemble mean temperature (thick solid lines) is modest, amounting to a $5 \%$ reduction of the centennial GMST change projected under RCP4.5, with VOLC and VOLC-CONST being slightly cooler than NO-VOLC throughout the post-2005 period, as expected from the first-order response to volcanic forcing. Near-term GMST projections for the $2016-2035$ period (Fig. 2b) exhibit only a small

\footnotetext{
${ }^{1}$ Uni Research Climate, Bjerknes Centre for Climate Research, Bergen 5007, Norway. ${ }^{2}$ Nansen Environmental and Remote Sensing Center, Bjerknes Centre for Climate Research, Bergen 5006, Norway. ${ }^{3}$ NCAS-Climate, Department of Meteorology, University of Reading, Reading RG6 6BB, UK. ${ }^{4}$ Institute for Coastal Research, Helmholtz-Zentrum Geesthacht, Geesthacht 21502, Germany. ${ }^{5}$ Laboratory of Environmental Chemistry, Paul Scherrer Institute, Villigen 5232, Switzerland. ${ }^{6}$ Oeschger Centre for Climate Change Research, University of Bern, Bern 3012, Switzerland. ${ }^{7}$ Irish Climate Analysis and Research Units, Department of Geography, National University of Ireland Maynooth, Maynooth, County Kildare, Ireland. *e-mail: ingo.bethke@uni.no
} 

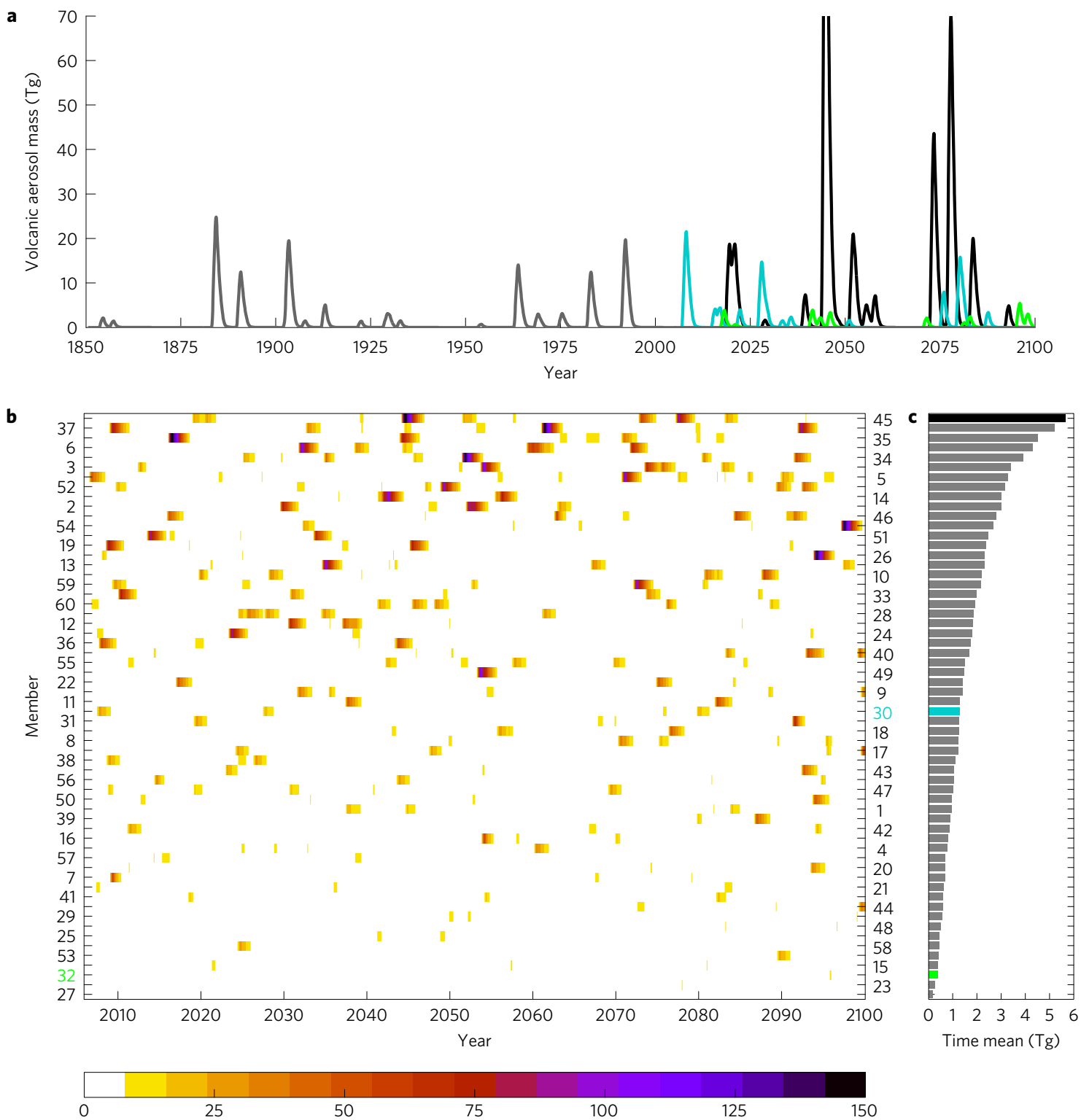

Figure 1 | Historical and plausible future volcanic forcing. a, Stratospheric volcanic aerosol loadings in the model's historical and twenty-first century CMIP5 simulations ${ }^{6,27}$ (grey) and synthetic forcing realizations with statistically low (5\%, green), mid (50\%, cyan) and extreme (>98\%, black) loadings when compared to the historical reconstructions over the past 2,500 years ${ }^{5}$. The data are monthly, filtered with a 12 -month running mean.

b,c, Stratospheric aerosol loading time series (b) and their century means for all simulation members (c). Members are ranked according to their time-mean loadings. Colour marked realizations correspond to the three realizations displayed in $\mathbf{a}$.

$(0.05 \mathrm{~K})$ reduction in mean response in VOLC and VOLC-CONST, with an increased skew in VOLC leading to a $0.1 \mathrm{~K}$ shift in the lower distribution tail. As a result, the $1.5^{\circ} \mathrm{C}$ warming target of the Paris agreement COP-21 (ref. 24) is exceeded on average two years later in VOLC and VOLC-CONST (Supplementary Table 1), with the upper distribution tail of VOLC being shifted by twice that amount (Fig. 2c). Models which did not include constant background forcing in their standard twenty-first century simulations prepared for CMIP5 are thus slightly overly pessimistic as to the likely time until different warming thresholds are reached.

Over the course of the simulation period, the ensemble mean difference grows, eventually saturating just below $0.1 \mathrm{~K}$ around 2040 (Fig. 2a), after which the means are well separated. The delay highlights the role of slow-response components, particularly the ocean ${ }^{16,17}$, in aggregating the global response to episodic volcanic forcing. The general correspondence of the VOLC and VOLC-CONST ensemble shows that the application of a time-invariant background forcing adequately accounts for long-term aspects of volcanic impacts in the ensemble mean projections ${ }^{28}$. One could use plausible low and high background values to further account for projection uncertainty stemming from uncertainty in the centennial-mean volcanic forcing (Fig. 1c and Supplementary Fig. 1). However, this would fail to capture the response to episodic volcanic forcing and attendant impacts on annual-to-decadal variability and extremes.

The interannual uncertainty range (5-95\% ensemble spread) in annual-mean GMST is inflated by more than $50 \%$ (from 0.3 to $0.5 \mathrm{~K}$ ) in VOLC relative to NO-VOLC (Fig. 2a-red versus blue shading; Supplementary Fig. 9 shows GMST from individual ensemble members). Consistent with a tropospheric cooling response, the change in ensemble spread in VOLC relative to NO-VOLC is skewed towards lower GMST, leaving the higher bound largely unaltered (Fig. 2d). Reductions in frequency of extremely warm years are generally small, whereas increases in frequency of extremely cold 
a
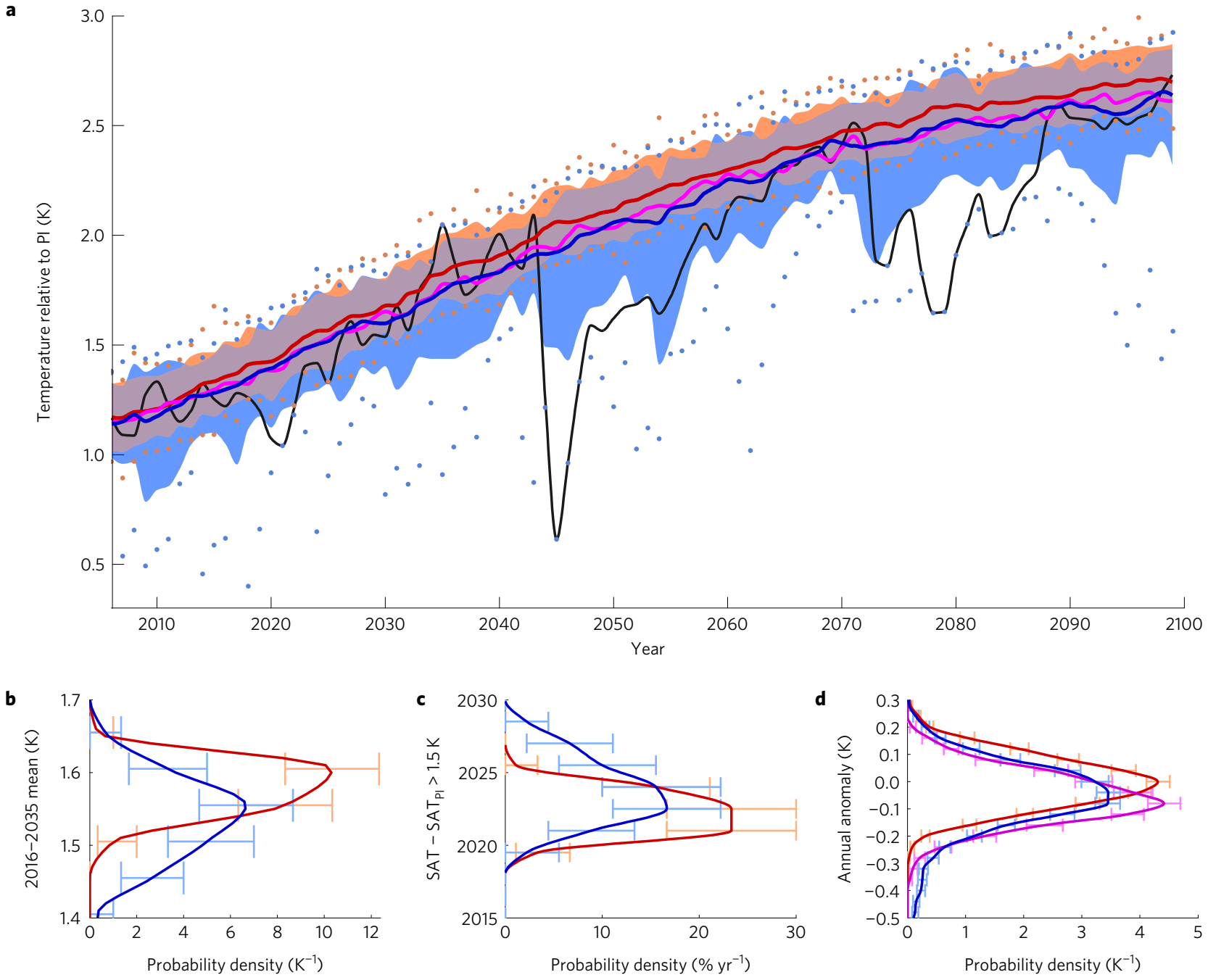

Figure 2 | Annual-mean GMST. a, Ensemble mean (solid) of VOLC (blue), VOLC-CONST (magenta) and NO-VOLC (red/orange) with 5-95\% range (shading) and ensemble minima/maxima (dots) for VOLC and NO-VOLC; evolution of the most extreme member (black). b, Probability density function (PDF) of the 2016-2035 mean relative to pre-industrial (PI, see Methods), with 5-95\% bootstrap confidence bounds. c, PDF of the time when SAT change relative to PI (20-year running average) exceeds $1.5 \mathrm{~K}$. d, PDF of annual anomalies with anthropogenic trend removed. The spread of VOLC-CONST is linearly shifted relative to NO-VOLC, and therefore not shown in $\mathbf{a}-\mathbf{c}$.

years-relative to the moving average or 'present-day' climate at any point-are much more substantial. In contrast, the application of a constant background forcing merely shifts the distribution of VOLC-CONST relative to NO-VOLC, overestimating the reduction of warm years and underestimating the increase of cold years.

Decadal-scale GMST series are even more affected by future volcanic forcing uncertainty than annual temperatures (Fig. 3a). The distribution of the decadal means-with the global warming trend removed prior to the analysis-is considerably wider for VOLC than for NO-VOLC, with roughly a doubling in standard deviation (Fig. $3 \mathrm{~b}$ and Supplementary Table 1). Anomalously cold decades become more frequent at the expense of 'normal' and, to a lesser degree, anomalously warm decades. As for decadal means, the spread in decadal trends is significantly wider for VOLC than for NO-VOLC (Fig. 3c). Occurrences of decades with negative GMST trend become more frequent if accounting for volcanic forcing, with the probability increasing from $10 \%$ in NO-VOLC to more than $16 \%$ in VOLC (Fig. 3d). Conversely, the widening of the upper tail of the decadal trend distribution (Fig. 3c) indicates enhanced probability of decadal-scale warming surges, due to the rebound of GMST after volcanic-induced cooling has reached its maximum (see Supplementary Fig. 4). The probability of decades with negative GMST trend more than doubles from $4 \%$ to $10 \%$ (Fig. 3e) if the analysis is limited to the first half of the century-before the stabilization period of RCP4.5-suggesting that the relative impact is sensitive to other forcings and depends on the anthropogenic scenario. Volcanic-induced cooling becomes increasingly important in facilitating neutral or negative temperature trends on longer timescales on which natural internal variability effects such as El Niño are no longer sufficient to offset anthropogenic forcings (Fig. 3f,g).

That volcanic influence is not limited to GMST projections becomes evident from assessing selected global and large-scale climate indicators that all have previously been found to be sensitive to volcanism ${ }^{13,17-20}$ (Fig. 4 and Supplementary Table 1). The radiative forcing at the top of the atmosphere is reduced by $0.05 \mathrm{~W} \mathrm{~m}^{-2}$ on average (Fig. 4a), whereas its decadal standard deviation, including the anthropogenic RCP 4.5 signal, is increased by $80 \%$ in response to volcanic forcing. The distribution of decadal radiative anomalies is widened, with a skew towards lower values (Fig. $4 \mathrm{~b}$ ) and a slight occurrence of more positive extremes resulting from reduced radiative surface cooling in post-eruption years. Global sea-level rise is on average slowed by $4 \%$ (relative to RCP4.5) in VOLC compared to NO-VOLC (Fig. 4c) as a direct consequence of reduced heat uptake by the oceans. The distribution of decadal sea level 

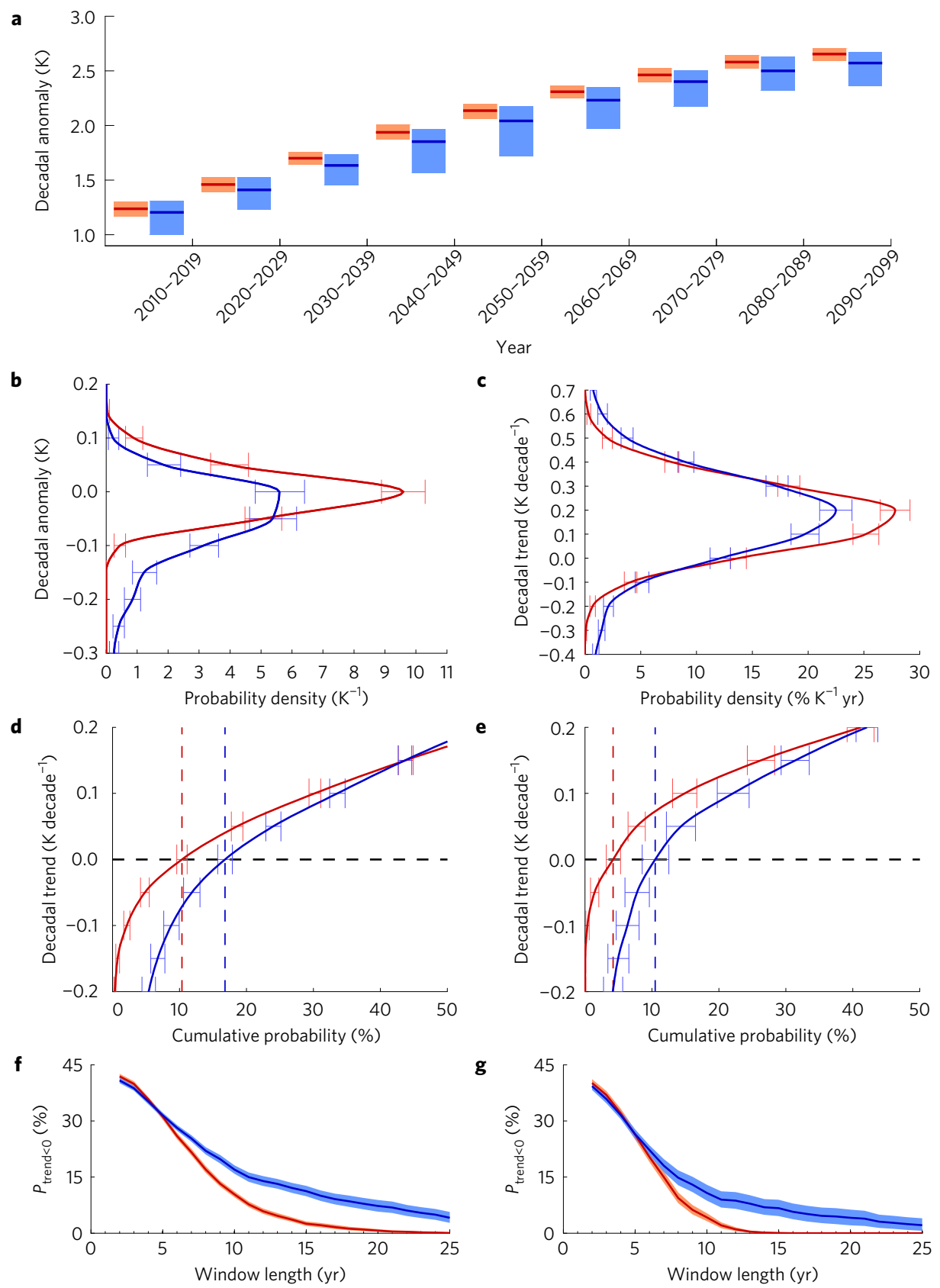

Figure 3 | Decadal temperature means and trends. a, Decadal means of GMST relative to pre-industrial. Ensemble mean (solid) with 5-95\% range (shading) of VOLC (blue) and NO-VOLC (red). b, PDF with 5-95\% bootstrap confidence bounds of decadal anomalies (without overlap) relative to NO-VOLC ensemble mean. c, As b, but for decadal trends. d, Cumulative probability distribution with 5-95\% confidence bounds for decadal trends (with overlap), using a 10-year window that is moved over 2006-2099. f, Probability for obtaining negative trends as function of length (solid) with 5-95\% bootstrap confidence bounds (shading). e,g, As d,f, but for the shorter period 2006-2050.

anomalies is significantly widened (doubling of standard deviation after subtracting global warming trend) with the lower uncertainty tail being affected most (Fig. 4d). Contrary to GMST, the volcanic forcing is generally not strong enough to halt global steric sea-level rise by offsetting anthropogenic-driven ocean warming on decadal and longer timescales. Asian summer monsoon precipitation shows consistent, albeit small reductions (Fig. 4e), with all decades featuring lower ensemble means, and a $20 \%$ overall increase in ensemble standard deviation (Fig. 4f). The Atlantic Meridional Overturning Circulation at $26^{\circ} \mathrm{N}$ shows a relative strengthening of $0.2 \mathrm{~Sv}$ in VOLC compared to NO-VOLC (Fig. $4 \mathrm{~g}$ ), with all decades exhibiting increased ensemble means, and a $20 \%$ overall increase in ensemble standard deviation (Fig. 4h). Similarly, Arctic sea-ice volume shows a $1-2 \%$ relative increase for most decades (Fig. 4i) and a $15 \%$ increase in ensemble standard deviation (Fig. 4j), with more overlap between the spread of subsequent decades in VOLC compared to NO-VOLC, indicating enhanced probability for a temporary halt in Arctic sea-ice decline.

To address if the inclusion of volcanic forcing variability has local implications we performed a time-of-emergence (ToE) analysis ${ }^{8}$ on seasonally averaged surface air temperature (Fig. 5). The ToE is formally defined as the mean time at which the signal of global warming emerges from the noise of natural climate variability (see Methods). The simulated impact of volcanic forcing variability on the ToE changes is distinct but small. The ToE is delayed almost everywhere as a consequence of the inclusion of volcanic forcing 


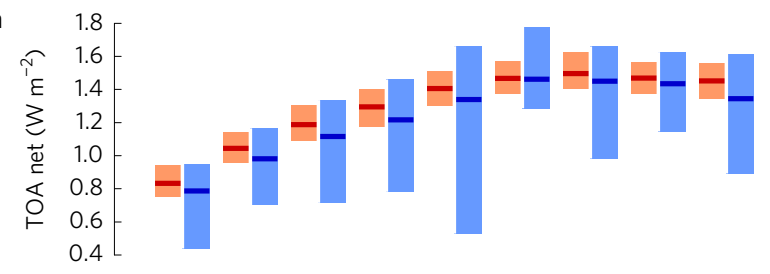

C

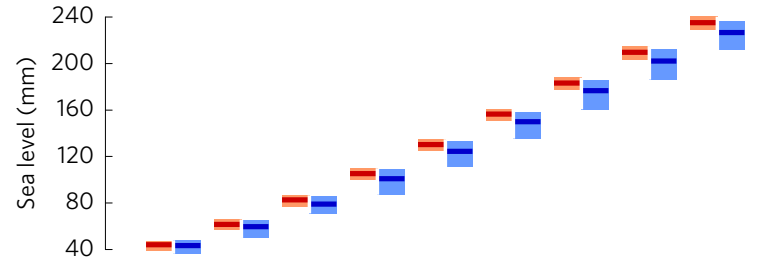

e

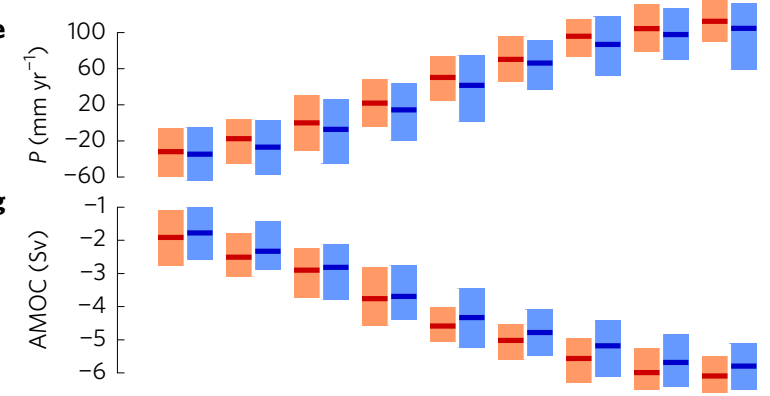

i

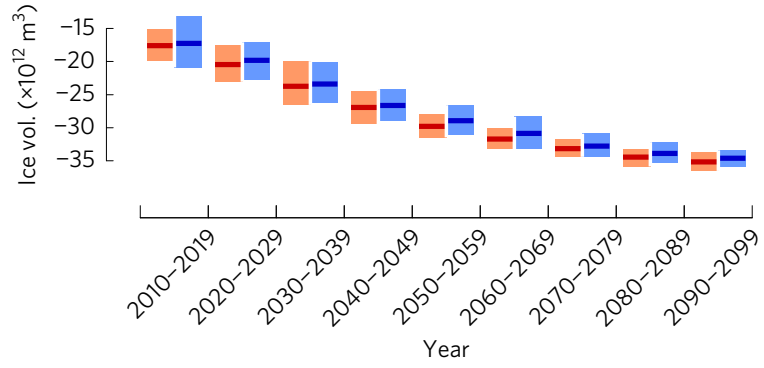

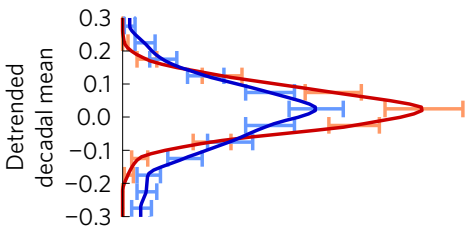

d
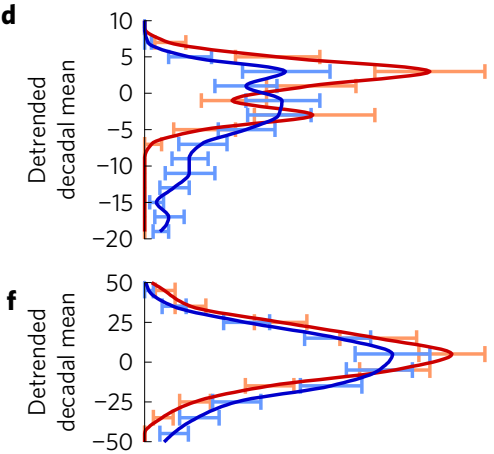

h

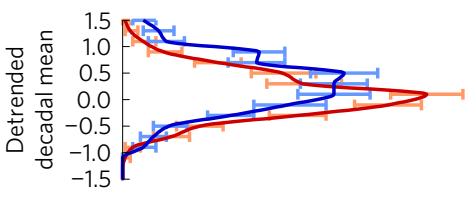

j

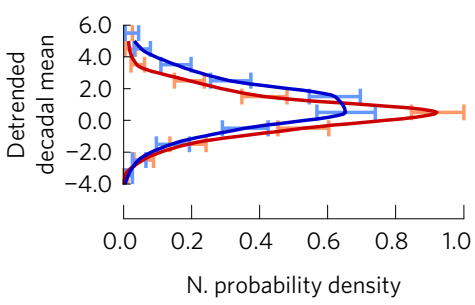

Figure 4 | Decadal means of large-scale climate indicators. a, Top-of-atmosphere net radiation balance. c, Global steric sea level. e, May-September precipitation, averaged over Asian continent box $\left(60^{\circ}-135^{\circ} \mathrm{E}, 5^{\circ}-55^{\circ} \mathrm{N}\right)$ (see Supplementary Fig. 5). g, Atlantic Meridional Overturning Circulation (AMOC) strength at $26^{\circ} \mathrm{N}$. i, Northern Hemisphere sea-ice volume. Ensemble mean relative to pre-industrial (solid) of VOLC (blue) and NO-VOLC (red)

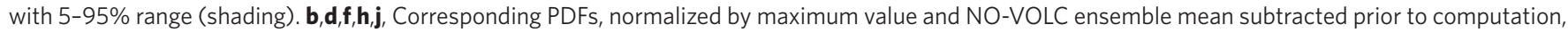
with 5-95\% bootstrap confidence bounds. The results of VOLC-CONST are shifted relative to NO-VOLC but otherwise similar and therefore not shown.

(Fig. 5a,b). The distribution of the simulated delay is strongly skewed, with delays of up to a decade in some locations, and of three years on average (Fig. 5 c,d).

Our results highlight the importance of representing volcanic forcing uncertainty in probabilistic future climate projections, in particular for risk assessments with focus on variability and certain extremes. Counter to earlier findings of destructive interference of volcanic forcing with internal climate variability modes ${ }^{18}$, our stochastic volcanic forcing generally amplifies the annual-todecadal scale climate variability in our model. A sharp increase in simulated decades with negative GMST trend exemplifies the effect of volcanic forcing uncertainty on projections of climate extremes with direct socio-economic consequences-such as Arctic sea-ice extent, monsoon precipitation, mid-latitude storminess and temperature. Extreme volcanic activity can potentially cause extended anomalously cold periods. This will, however, not help to mitigate long-term global warming impacts as the surface climate is likely to rebound, leaving its long-term trajectory unaltered (Fig. 2a, black curve).

This study is just a step towards incorporating current knowledge on global volcanic activity in probabilistic future climate projections in realistic and systematic ways. It serves as a proof of concept for a statistical representation of potential volcanism in twenty-first century climate projections and demonstrates the importance of such a representation for the projections of future climate variability. Our ensemble analysis, based on a single model and a single anthropogenic scenario, provides only a conditional assessment of the volcanic contribution to climate projection uncertainty ${ }^{29}$. Additional uncertainties in the volcanic forcing reconstruction, in other external forcings, and in the model warrant further experiments. Our simulations may still underestimate future volcanic impact due to a too low sensitivity to volcanic forcing and the omission of small eruptions that cannot be detected in ice cores. The relative impacts further depend on the simulated global warming and amount of unforced variability in the model (details in Supplementary Information). Since simulated regional impacts are less distinct than global impacts, and have larger model uncertainty ${ }^{29}$, quantifying volcanic impacts on regional climate projections and their socioeconomic aspects should be a priority of future work. Improved characterization of past volcanic forcing, improved representation of volcanic impact in models, and coordinated multi-model efforts using the same plausible forcings are essential ingredients for advancing the utilization of volcanism information in future climate assessments. The newly established Model Intercomparison Project on climatic response to Volcanic forcing (VolMIP) ${ }^{30}$ presents the ideal platform for integrating these efforts. 


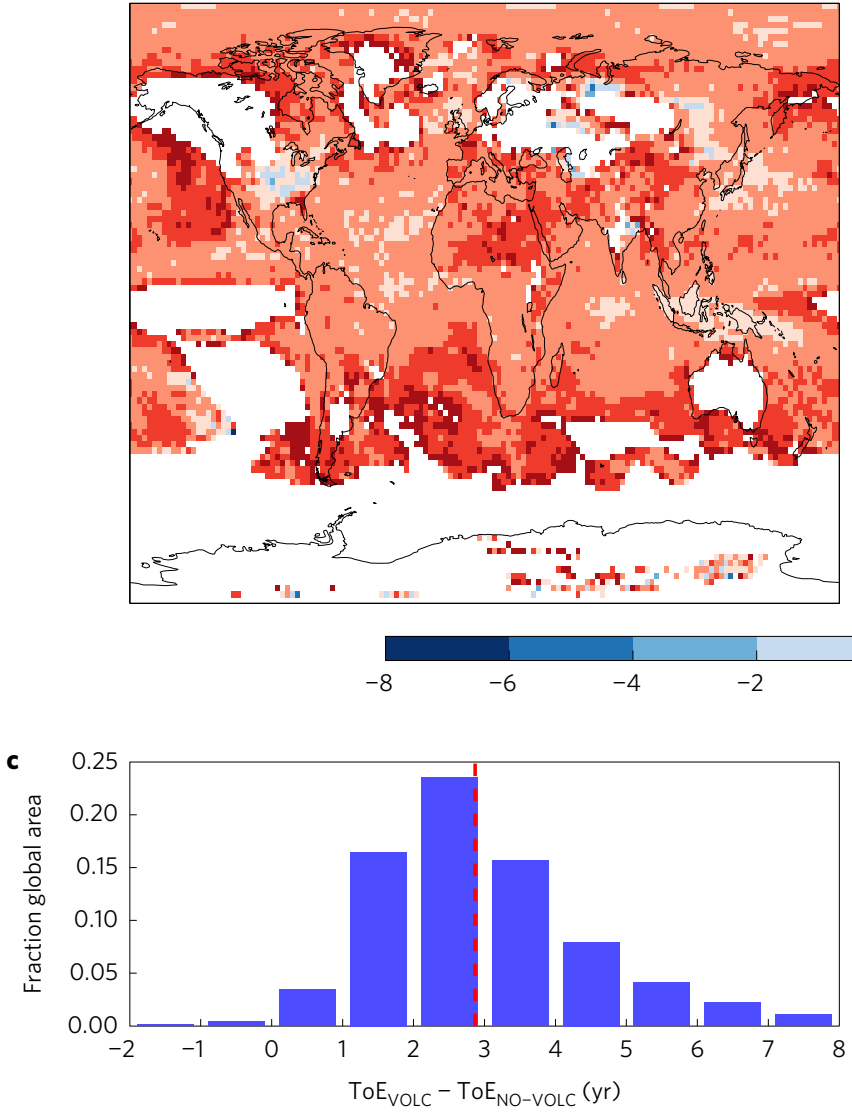

b

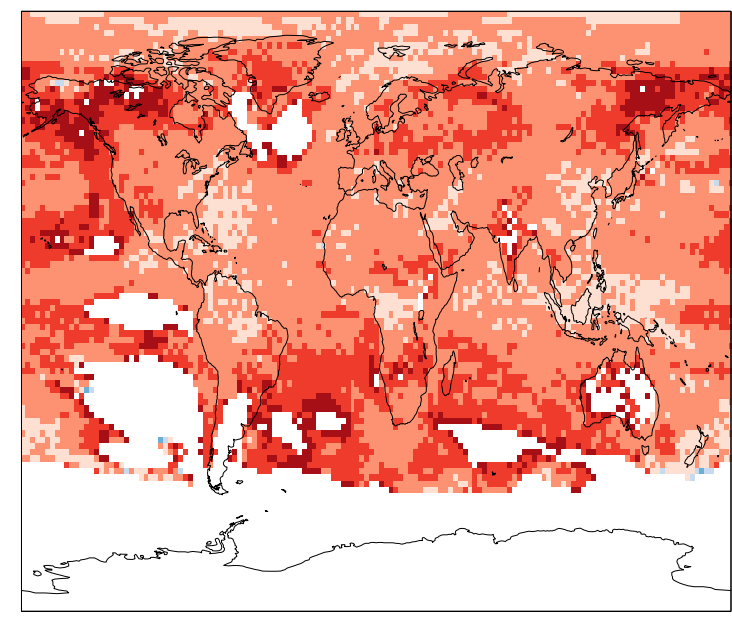

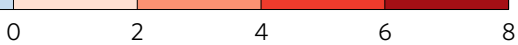

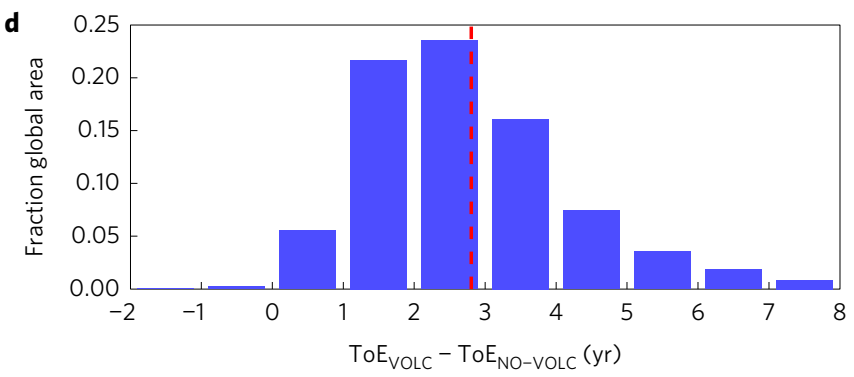

Figure 5 | Time of emergence of anthropogenic GMST changes. a,b, VOLC-NO-VOLC difference in ToE relative to 1985-2005 mean for boreal winter (a) and boreal summer (b) in years. Regions with no apparent emergence before 2100 are left blank. $\mathbf{c}, \mathbf{d}$, Areal distribution of ToE differences for boreal winter (c) and boreal summer (d), with dashed red lines denoting the corresponding weighted global means. The analysis includes only regions that show emergence between 2006 and 2099 in both ensembles.

\section{Methods}

Methods, including statements of data availability and any associated accession codes and references, are available in the online version of this paper.

\section{Received 8 March 2017; accepted 24 August 2017;} published online 2 October 2017

\section{References}

1. Schurer, A. P., Tett, S. F. B. \& Hegerl, G. C. Small influence of solar variability on climate over the past millennium. Nat. Geosci. 7, 104-108 (2014).

2. Kirtman, B. et al. in Climate Change 2013: The Physical Science Basis (eds Stocker, T. F. et al.) 953-1028 (IPCC, Cambridge Univ. Press, 2013).

3. Taylor, K. E. et al. An overview of CMIP5 and the experiment design. Bull. Am. Meteorol. Soc. 93, 485-498 (2012)

4. O'Neill, B. C. et al. The Scenario Model Intercomparison Project (ScenarioMIP) for CMIP6. Geosci. Model Dev. 9, 3461-3482 (2016).

5. Sigl, M. et al. Timing and climate forcing of volcanic eruptions for the past 2,500 years. Nature 523, 543-549 (2015).

6. Bentsen, M. et al. The Norwegian Earth System Model, NorESM1-M-Part 1: description and basic evaluation of the physical climate. Geosci. Model Dev. 6, 687-720 (2013).

7. Vuuren, D. P. et al. The representative concentration pathways: an overview. Climatic Change 109, 5-31 (2011).

8. Hawkins, E. \& Sutton, R. Time of emergence of climate signals. Geophys. Res Lett. 39, L01702 (2012).

9. Hyde, W. T. \& Crowley, T. J. Probability of future climatically significant volcanic eruptions. J. Clim. 13, 1445-1450 (2000).

10. Parker, D. E. \& Brownscombe, J. L. Stratospheric warming following the El Chichón volcanic eruption. Nature 301, 406-408 (1983).

11. Robock, A. \& Mao, J. The volcanic signal in surface temperature observations. J. Clim. 8, 1086-1103 (1995).
12. Iles, C. E. \& Hegerl, G. C. Systematic change in global patterns of streamflow following volcanic eruptions. Nat. Geosci. 8, 838-842 (2015).

13. Liu, F. et al. Global monsoon precipitation responses to large volcanic eruptions. Sci. Rep. 6, 24331 (2016).

14. Shindell, D. T., Schmidt, G. A., Mann, M. E. \& Faluvegi, G. Dynamic winter climate response to large tropical volcanic eruptions since 1600. J. Geophys. Res. 109, D05104 (2004).

15. Miller, G. H. et al. Abrupt onset of the Little Ice Age triggered by volcanism and sustained by sea-ice/ocean feedbacks. Geophys. Res. Lett. 39, L02708 (2012).

16. Gleckler, P. J. et al. Volcanoes and climate: Krakatoa's signature persists in the ocean. Nature 439, 675 (2006).

17. Stenchikov, G. et al. Volcanic signals in oceans. J. Geophys. Res. 114, D16104 (2009).

18. Swingedouw, D. et al. Bidecadal North Atlantic ocean circulation variability controlled by timing of volcanic eruptions. Nat. Commun. 6, 6545 (2015).

19. Otterå, O. H., Bentsen, M., Drange, H. \& Suo, L. External forcing as a metronome for Atlantic multidecadal variability. Nat. Geosci. 3, 688-694 (2010)

20. Church, J. A., White, N. J. \& Arblaster, J. M. Significant decadal-scale impact of volcanic eruptions on sea level and ocean heat content. Nature 438, 74-77 (2005).

21. Hansen, J. et al. Global climate changes as forecast by Goddard Institute for Space Studies three-dimensional model. J. Geophys. Res. 93, 9341-9364 (1988).

22. Shiogama, H. et al. Possible influence of volcanic activity on the decadal potential predictability of the natural variability in near-term climate predictions. Adv. Meteorol. 2010, 657318 (2010).

23. Ammann, C. M. \& Naveau, P. A statistical volcanic forcing scenario generator for climate simulations. J. Geophys. Res. 115, D05107 (2010).

24. Mitchell, D. et al. Realizing the impacts of a $1.5^{\circ} \mathrm{C}$ warmer world. Nat. Clim. Change 6, 735-737 (2016).

25. Self, S. \& Gertisser, R. Tying down eruption risk. Nat. Geosci. 8, 248-250 (2015).

26. Tuel, A., Naveau, P. \& Ammann, C. M. Skillful prediction of multidecadal variations in volcanic forcing. Geophys. Res. Lett. 44, 2868-2874 (2017). 
27. Ammann, C. M., Meehl, G. A., Washington, W. M. \& Zender, C. S. A monthly and latitudinally varying volcanic forcing dataset in simulations of 20th century climate. Geophys. Res. Lett. 30, 1657 (2003).

28. Gregory, J. M. Long-term effect of volcanic forcing on ocean heat content. Geophys. Res. Lett. 37, 045507 (2010).

29. Hawkins, E. \& Sutton, R. The potential to narrow uncertainty in regional climate predictions. Bull. Am. Meteorol. Soc. 90, 1095-1107 (2009).

30. Zanchettin, D. et al. The Model Intercomparison Project on the climatic response to Volcanic forcing (VolMIP): experimental design and forcing input data for CMIP6. Geosci. Model Dev. 9, 2701-2719 (2016).

\section{Acknowledgements}

We thank D. Mitchell, A. Kirkevåg, M. Toohey and D. Zanchettin for providing valuable input. This study was supported by the Bjerknes Centre for Climate Research, Research Council of Norway (229774) and UNINETT Sigma2 (nn9039k, ns9039k).

\section{Author contributions}

S.O., P.T. and I.B. developed the stochastic forcing model. S.W. and M.S. helped with the utilization and interpretation of the ice-core reconstructions. I.B., P.T., S.O. and E.H conceived and designed the simulation experiments. E.H. performed the ToE analysis. All authors contributed to writing the manuscript.

\section{Additional information}

Supplementary information is available in the online version of the paper. Reprints and permissions information is available online at www.nature.com/reprints. Publisher's note: Springer Nature remains neutral with regard to jurisdictional claims in published maps and institutional affiliations. Correspondence and requests for materials should be addressed to I.B.

\section{Competing financial interests}

The authors declare no competing financial interests. 


\section{Methods}

Historical volcanic data utilized. We consider multiple ice cores from Greenland and Antarctica utilizing the sulfate contained in the ice core as a proxy for explosive volcanic activity of the past 2,500 years ${ }^{5}$. Although the approach is subject to uncertainties arising from issues such as dating uncertainties, scaling of sulfate peaks to changes in stratospheric aerosol loads, representativeness of different regions over Greenland and Antarctica for volcanic sulfate emissions, and discrimination of single tropical versus two individual quasi-contemporaneous high-latitude eruptions, it provides an insight into the plausible range of magnitude and temporal structure of future volcanic eruptions. Ice-core records, however, do omit small volcanic events which may nevertheless have some impacts on climate $^{31}$. Earlier reconstructions $\mathrm{s}^{32-34}$ of past volcanic eruptions for the past millennium show similar temporal evolution and timing, albeit with differences in magnitude. The use of multiple cores in our reconstruction is thought to improve on earlier overestimates of, in particular, larger eruptions ${ }^{35}$ such as the Samalas eruption in 1257 (ref. 36). The series represents the longest currently available annually resolved continuous series, making it particularly suitable for our study. The series provides information on timing, magnitude of sulfur injection, and location (tropical versus extratropical in respective hemisphere) of a total of 283 eruption events for which ice-core sulfate concentrations exceeded a detection threshold (approximately $1 / 3 \mathrm{rd}$ of the strength of a tropical eruption such as Pinatubo in 1991) defined by the natural variability of non-volcanic sulfate in the ice. Eruptions from Iceland and Alaska are expected to be overrepresented in this data set due to their proximity to Greenland. Anthropogenic $\mathrm{SO}_{2}$ emissions predominantly from the United States and Europe peaking in the 1970-1980s (that is, 'global dimming ${ }^{37}$ ) mask volcanic sulfate contributions in Greenland ice cores during parts of the twentieth century ${ }^{38}$, hampering detection and quantification of volcanic sulfate deposition for eruptions such as El Chichón in 1982. Nevertheless, that the past 150 years were comparatively 'quiet' is supported by the ice cores from Antarctica, which are not subject to significant human sulfate pollution and suggest that the stratospheric loadings between 1850 and 2000 CE were 30\% lower compared to the $1-2000 \mathrm{CE}$ average ${ }^{35}$

Stochastic forcing generation. We generated plausible future eruption chronologies by resampling the past volcanic activity described in the prior section (code in Supplementary Information). For each month of the period 2006 to 2099, we draw random numbers from a uniform distribution to test whether one or more of the 283 eruption events in Sigl et al. ${ }^{5}$ were triggered in that ensemble member on that date. For the test we assumed a constant eruption probability of $1 /(2,500 \times 12)$, which is the probability of randomly picking the exact month from the 2,500-year record when a specific eruption event occurred. By repeating this procedure for all months and all ensemble members, we obtained 60 unique plausible future eruption chronologies for the twenty-first century (using the CMIP5 standard period 2006-2099), with statistics that resemble those of the historical reconstruction (Supplementary Fig. 2). We estimated peak stratospheric sulfate aerosol loadings by scaling the ice-core depositions of the individual events using established scaling relations ${ }^{32}$.

Implementation and validation of volcanic forcing. The ice-core-based reconstruction of Sigl et al..$^{5}$ provides global estimates for maximum stratospheric volcanic aerosol load following the volcanic eruptions and whether the eruptions were tropical or $\mathrm{NH} / \mathrm{SH}$ extratropical. To translate this information into model forcing, we analysed the data set of Ammann et al. ${ }^{27}$ who used a transport model to estimate the spatio-temporal dispersal of stratospheric volcanic aerosols for the twentieth century.

We found that their dispersal evolution can be approximated by three shape functions (Supplementary Fig. 6) - one for tropical and two for extratropical $\mathrm{NH} / \mathrm{SH}$ eruptions - that depend only on pressure, latitude and time since eruption start. We derived the final model forcing by scaling the shape functions with the maximum aerosol load estimated from the ice-core reconstruction. Our forcing implementation thus does not consider the seasonal effects on dispersal of volcanic aerosols discussed in Ammann and colleagues ${ }^{27}$.

We compare ice-core-based historical forcing, that we constructed in the same way, to the Ammann et al. ${ }^{27}$ forcing for the period 1850-2000 to assess whether our model forcing is biased towards low or high values (Supplementary Fig. 7; details in Section 2 in Supplementary Information). Although the timing and forcing magnitudes match well for some eruptions (for example, 1883-Krakatau, 1963-Agung and 1991-Mt. Pinatubo), they differ for others (for example, 1902-Santa Maria, 1980-Mt. St. Helens and 1982-El Chichón) partly for reasons previously outlined. Despite discrepancies for individual eruptions, however, no systematic differences in size or frequency distributions are seen and the time means- $1.15 \mathrm{Tg}$ versus $1.22 \mathrm{Tg}$ volcanic aerosol in ice-core-based versus Ammann et al. ${ }^{27}$ forcing-show differences of less than $10 \%$.

Model configuration. We performed all simulations with the medium resolution configuration of the Norwegian Earth System Model version 1 (NorESM1-M) (refs 6,39), a state-of-the-art climate model that provided input to the Fifth Coupled Model Intercomparison Project (CMIP5) (ref. 3). We used the exact configuration that was employed in performing those CMIP5 runs. NorESM1-M is based on the Community Climate System Model version 4 (ref. 40). Important modifications to the latter are the employment of an isopycnic coordinate ocean component, improving the conservation and transformation properties of water masses, and the addition of a more advanced aerosol-cloud chemistry treatment in the atmosphere component. The land and sea-ice components are adopted in their original form.

The atmosphere and land components are configured on a regular $1.9^{\circ} \times 2.5^{\circ}$ horizontal grid, whereas the ocean and sea-ice components are configured on a $1^{\circ}$ curvilinear horizontal grid with the northern pole singularity shifted over Greenland. The atmospheric component features 26 hybrid sigma-pressure levels extending to $3 \mathrm{hPa}$. The ocean component features a stack of 51 isopycnic layers, with a variable depth bulk mixed layer on top.

NorESM1-M has been used to study the effect of major extratropical eruptions ${ }^{41,42}$, where volcanic sulfur dioxide was directly injected into the atmosphere and subsequent oxidation to sulfate aerosols was simulated by the model. This approach is not applicable here as it would require a statistical model for sulfur emissions that can provide exact geographic locations, injection rates as well as injection heights. Hence, we prescribe volcanic sulfate aerosol concentrations, following the approach used for NorESM's CMIP5 simulations ${ }^{6}$, where the model reads a mass distribution of stratospheric volcanic sulfate aerosols (Supplementary Fig. 6), which is converted to number concentrations-assuming a fixed, log-normal size distribution - and combined with prognostic sulfate before being passed to the radiation code.

Model spin-up and sensitivity test. We generated starting conditions for the twenty-first century simulations by performing a 26-year spin-up experiment, which we spawned off three simulation sets with 20 members each from the year 1980 states of NorESM's three historical CMIP5 simulations ${ }^{6}$. Initial spread within each set was generated through adding small $\left(O\left(10^{-6} \mathrm{~K}\right)\right)$ noise to the ocean mixed-layer temperatures. The total of 60 members were integrated to the end of year 2005, after which time the ensemble spread had reached saturation on most relevant diagnostics ${ }^{43,44}$, such as AMOC variability (Supplementary Fig. 8). As a result, the covariability between individual members of our twenty-first century simulations is very close to zero.

We performed a 60-member spin-up ensemble for the post-Mt. Pinatubo eruption period 1990-2005, that is identical to the first one but with the forcing of the Mt. Pinatubo eruption removed. Analysing the differences of the two ensembles allowed us to verify the model's sensitivity to volcanic forcing (details in Supplementary Information).

Bootstrap confidence intervals. We used empirical bootstrapping ${ }^{45}$ to assess statistical robustness. For each test, we generated 10,000 bootstrap samples by resampling the original data with replacement. We then performed our analysis on each bootstrap sample and derived 5-95\% confidence intervals by ranking the results. In the generation of the bootstrap sample, we treated the data from individual simulations as contiguous blocks ${ }^{46}$ to account for effects of autocorrelation along the temporal dimension. The autocorrelation along the ensemble dimension was zero by construction.

Pre-industrial (PI) reference climate. We show all diagnostics relative to a pre-industrial reference climate to put the volcanic impacts into a climate-change perspective. We computed the reference climatology from the 500-year-long pre-industrial control simulation that NorESM contributed to CMIP5 (ref. 6). This simulation used external forcings fixed at 1850 levels and no volcanic forcing.

Time-of-Emergence (ToE) analysis. Our ToE analysis on seasonally averaged surface air temperature follows Hawkins and Sutton ${ }^{8}$. We define ToE as the earliest occurrence where the signal-to-noise ratio exceeds the value 2 (climate-change signal is distinguishable from zero at a $95 \%$ confidence level). For each simulation member, we estimated the signal by regressing the local time series onto the corresponding global-mean time series. We then estimated the noise, that is, unforced internal climate variability, from standard deviations of the aforementioned 500-year-long pre-industrial control simulation ${ }^{6}$ that was run with the same model configuration as used in this study. The obtained ToEs were averaged over the respective NO-VOLC and VOLC ensemble. The analysis was performed for extended boreal winter (October-March) and boreal summer (April-September) averages.

Code availability. The code for the generation of synthetic volcanic forcings is included in the Supplementary Information. The Norwegian Earth System Model can be obtained by sending a request to noresm-ncc@met.no.

Data availability. The model output from this study and volcanic forcing ancillaries are available at https://doi.org/10.11582/2017.00006. 


\section{References}

31. Santer, B. D. et al. Volcanic contribution to decadal changes in tropospheric temperature. Nat. Geosci. 7, 185-189 (2014).

32. Gao, C., Oman, L., Robock, A. \& Stenchikov, G. L. Atmospheric volcanic loading derived from bipolar ice cores: accounting for the spatial distribution of volcanic deposition. J. Geophys. Res. 112, D09109 (2007).

33. Crowley, T. J. \& Unterman, M. B. Technical details concerning development of a $1200 \mathrm{yr}$ proxy index for global volcanism. Earth Syst. Sci. Data 5, 187-197 (2013).

34. Sigl, M. et al. A new bipolar ice core record of volcanism from WAIS Divide and NEEM and implications for climate forcing of the last 2000 years. J. Geophys. Res. 118, 1151-1169 (2013).

35. Sigl, M. et al. Insights from Antarctica on volcanic forcing during the Common Era. Nat. Clim. Change 4, 6-10 (2014)

36. Vidal, C. M. et al. The 1257 Samalas eruption (Lombok, Indonesia): the single greatest stratospheric gas release of the Common Era. Sci. Rep. 6, 34868 (2016).

37. Wild, M. et al. From dimming to brightening: decadal changes in Solar radiation at Earth's surface. Science 308, 847-850 (2005).

38. McConnell, J. R. et al. 20th-century industrial black carbon emissions altered Arctic climate forcing. Science 317, 1381-1384 (2007).
39. Iversen, T. et al. The Norwegian Earth System Model, NorESM1-M-Part 2: climate response and scenario projections. Geosci. Model Dev. 6, 389-415 (2013).

40. Gent, P. R. et al. The community climate system model version 4. J. Clim. 24, 4973-4991 (2011).

41. Pausata, F. S. R., Grini, A., Caballero, R., Hannachi, A. \& Seland, Ø. High-latitude volcanic eruptions in the Norwegian Earth System Model: the effect of different initial conditions and of the ensemble size. Tellus B 67, 26728 (2015).

42. Pausata, F. S. R., Chafik, L., Caballero, R. \& Battisti, D. S. Impacts of high-latitude volcanic eruptions on ENSO and AMOC. Proc. Natl Acad. Sci. USA 112, 13784-13788 (2015).

43. Outten, S., Thorne, P., Bethke, I. \& Seland, Ø. Investigating the recent apparent hiatus in surface temperature increases: 1 . Construction of two 30-member Earth System Model ensembles. J. Geophys. Res. 120, 8575-8596 (2015).

44. Thorne, P., Outten, S., Bethke, I. \& Seland, Ø. Investigating the recent apparent hiatus in surface temperature increases: 2 . Comparison of model ensembles to observational estimates. J. Geophys. Res. 120, 8597-8620 (2015).

45. Efron, B. \& Tibshirani, R. Bootstrap methods for standard errors, confidence intervals, and other measures of statistical accuracy. Stat. Sci. 1, 54-75 (1986).

46. Carlstein, E. The use of subseries values for estimating the variance of a general statistic from a stationary sequence. Ann. Stat. 14, 1171-1179 (1986). 